zirrhose. Wien. klin. Wschr, 78, (im Druck) (1966b). Searcy, R. L., L. M. Bergquist, and R. C. JUNG: Rapid ultramicro estimation of serum total cholesterol. J. Lipid. Res. 1, 349-351 (1960). - Trout, D. L., E. H. Estes jr., and S. J. FrIEDBera: Titration of free fatty acids of plasma: A study of current methods and a new modification. J. Lipid Res. 1, 199-202 (1960).

Prof. Dr. H. Braunsteiner

Med. Univ.-Klinik

A 6010 Innsbruck, Österreich

\title{
ACTH-Bestimmungen im Plasma aus dem Bulbus cranialis venae jugularis*
}

\author{
P. C. Scrtba, R. Hacker, P. Dieterle, F. Kluge, W. Hochmouser und K. Sohwarz \\ II. Medizinische Klinik der Universität München (Direktor: Prof. Dr. Dr. G. Bodechtel)
}

Das venöse Blut gelangt aus dem Hypophysenvorderlappen im wesentlichen über den Sinus cavernosus und die Sinus petrosi in die Bulbi craniales der beiden Venae jugulares. Ausgangspunkt für unsere Untersuchungen war die Überlegung, daß man im Bulbus cranialis venae jugularis höhere ACTH-Aktivitäten erwarten sollte als in der Peripherie (Vena cubitalis). Der Bulbus cranialis venae jugularis ist beim Menschen relativ einfach zu punktieren, wie das z. B. zur Messung der Hirndurchblutung üblich ist $[1,2]$. Es war zu klären, ob die mangelnde Empfindlichkeit und die relative Ungenauigkeit, welche die biologische Bestimmung von ACTH-Spiegeln im nativen peripheren Plasma immer noch belastet, durch die zu erwartende größere Höhe des ACTH-Gehalts im Plasma aus dem Bulbus cranialis venae jugularis auszugleichen wären.

\section{Methoden und Präparate}

Die biologische Bestimmung ron ACTH erfolgte mittels der von LTPSCOMB und NeLsoN angegebenen Methode [3] mit einigen Modifikationen anderer Autoren [4-6].

Männliche Sprague-Dawley-Ratten (190-230g) wurden auf parapharyngealem Wege in Avertin ${ }^{\circledR}$-Narkose hypophysektomiert [7-10]. Die Vollständigkeit der Hypophysektomie wurde nach jeder ACTH-Bestimmung in üblicher Weise durch Inspektion der Schädelbasis von oben verifiziert. - In Urethan $\left({ }^{\circledR}\right.$-Narkose wurde zuerst von der linken Fossa inguinalis aus ein Katheter in die Aorta thoracica geschoben, dann von der linken Vena femoralis aus nach Laparotomie die linke Nebennierenvene schlüssig katheterisiert und schließlich ein Katheter in die rechte Vena iliaca geschoben (Abb. 1). Nach den ACTH-Bestimmungen wurde die Lage der Katheter kontrolliert. Der Aortenkatheter lag sicher oberhalb des Abganges der Nebennierenarterien [11]. Alle ACTH-Gaben erfolgten über diesen Aortenkatheter, um die Empfindlichkeit der Methode gegenüber der intravenösen Injektion zu steigern. Die Tiere waren heparinisiert (50 E Heparin/100 g Ratte). Da nach der Hypophysektomie die Empfindlichkeit der Nebennierenrinde gegenüber ACTH-Gaben innerhalb von Stunden abnimmt $[12,13]$, wurden alle $A C T H-B e s t i m m u n g e n ~ 90 \mathrm{~min}$ nach Hypophysektomie begonnen, denn zu diesem Zeitpunkt war die Corticosteroninkretion in jedem Fall auf eine Basisinkretion abgesunken $[8-10]$.

Der Corticosterongehalt des aus der Nebennierenvene der Ratte stammenden Plasmas wurde fluorimetrisch nach ZFNKER und Bernstetry [14], modifiziert nach PFeiffer u. Mitarb. [4, $15]$ bestimmt. Doppelwerte der Plasmaproben wurden in zwei Reihen von Einzelwerten in der Reihenfolge der Extraktion gemessen $[8,9]$. AuBerdem wurden die sog. 11-Hydroxy. corticosteroide im menschlichen Plasma fluorimetrisch bestimmt $[16-18]$.

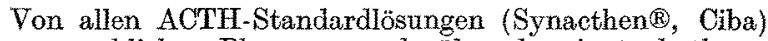
oder menschlichen Plasmen wurde uber den Aortenkatheter (Abb. 1) innerhalb von 94 sec jeweils genau $1 \mathrm{ml}$ infundiert (Perfusor, Fa, Braum, Melsungen), da die Corticosteroninkretion der Rattennebeniere bei konstantern ACTH-Spiegel von der Nebennierendurchblutung abhängt $[19,20]$. Größere Plasmavolumina [21-23] steigern die Nebennierendurch-

* Mit Unterstützung der Deutschen Forschungsgemeinschaft, der Stiftung VW-Werk (R. H.), der CB-Stiftung und der F. Baur-Stiftung. blutung erheblich. Die Wirkung von gleichen ACTH-Dosen ist aber nur bei möglichst gleicher Nebennierendurchblutung zu beurteilen.

Nach Vorversuchen $[8,10]$ wurde von der 3 . bis zur 11. min nach Beginn der ACTH-Infusion, bzw. der Infusion des zu untersuchenden Plasmas, Blut aus der Nebennierenvene der Ratte gesammelt und davon der Plasma-Corticosterongehalt bestimmt. Diese relativ lange Sammelperiode

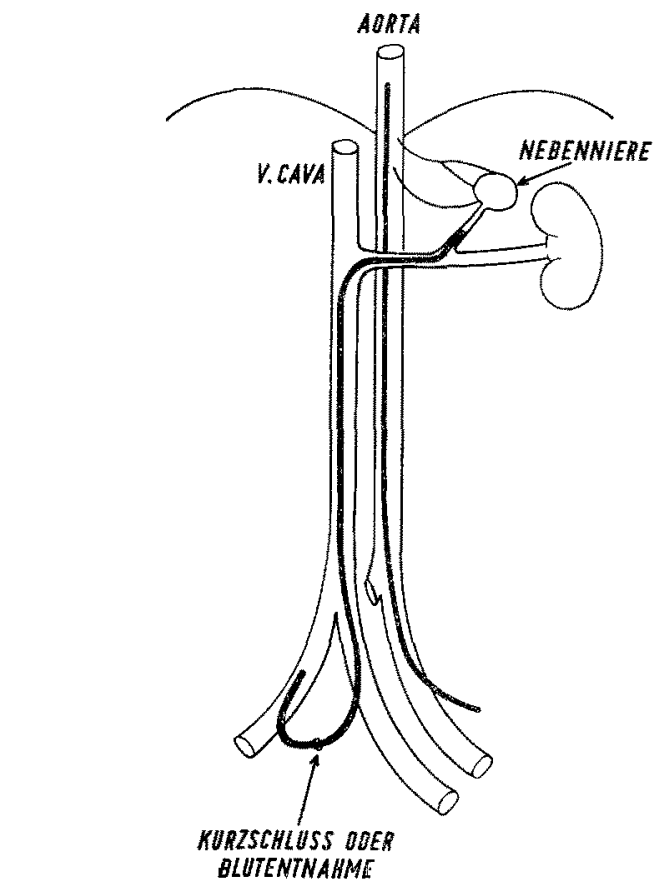

Abb. 1. Schema der Aorten- und Venenkatheterisation der Ratte. Die technische Durchführung der Katheterisationen ist unter Methoden besehrieben

garantiert, daß bei kleinsten ACTH-Dosen die Steigerung der Corticosteroninkretion vollständig erfaßt wird. Nach höheren ACTH-Dosen wird das Maximum der Steigerung der Corticosteroninkretion etwas später erreicht $[8,10,12]$. Eine Sammelperiode von $8 \mathrm{~min}$ erfaßt also nur eimen in Äbhängigkeit von der Dosis ACTH wechselnd kleinen Teil der Steigerung der Corticosteroninkretion $[8,10]$. Bei dieser Sammelperiode fand sich eine lineare Beziehung zwischen den logarithmisch aufgetragenen ACTH-Dosen und der ebenfalls logarithmiseh aufgetragenen Steigerung der Corticosteroninkretion pro $8 \mathrm{~min}$ (Abb. 2), welche nach Abzug der Basisfluorescenz der gemessenen Plasmavolumina als Differenz der Corticosteroninkretion pro $8 \mathrm{~min}$ vor und nach ACTH-Infusion ermittelt wurde. Diese lineare Kurve wurde erst oberhalb von $1,0 \mathrm{mE}$ ACTH flacher. Der Regressionskoeffizient der Eichkurve $(0,01-0,5 \mathrm{mE}$ ACTH) betrug $b=0,75[8-10,24]$, der Index der Genauigkeit $[6,25]$ der Methode war $\lambda=0,07$. Der Korrelationskoeffizient, $r=0,421$, war bei einer Irrtumswahrscheinlichkeit von 0,001 signifikant [24] von 0 verschieden. - Die Prüfung der Empfindlichkeit der Methode zeigte, daß zwischen der Dosis $0,01 \mathrm{mE}$ ACTH $(n=95)$ und einer NaCl-Infusion $(n=10)$ ein kaum signifikanter [24] Unterschied bestand $(0,15>p>0,10)$. Dagegen fand sich eine signifikante Differenz zwischen der Wirkung von $0,025 \mathrm{mE}$ ACTH $(n=23)$ und $0,01 \mathrm{mE}$ ACTH $(p<0,0025)$ sowie zwischen der Wirkung von $0,025 \mathrm{mE}$ ACTH und einer NaCl-Infusion $(p<0,01)$. Die 
Empfindlichkeitsgrenze der Methode liegt also zwischen 0,10 and $0,015 \mathrm{mE}$ ACTH.

Vor jeder Bestimmung eines AOTH-Plasmaspiegels korrekter ist die Bezeichnung ACTH-Gehalt im Plasma wurde der Ratte $1 \mathrm{ml}$ einer ACTH-Standardlösung $(0,05 \mathrm{mE}$ ACTH) infundiert. $20 \mathrm{~min}$ später war die Corticosteroninkretion wieder auf den Basiswert abgesunken, wie durch Blutentnahme aus der Nebennierenvene von der 20.-24. min kontrolliert wurde $[8,10]$. Darauf konnte dann das zu untersuchende Plasma infundiert und die Steigerung der Corticosteroninkretion pro $8 \mathrm{~min}$ wie oben gemessen werden. Dieses Vorgehen erlaubt eine Berücksichtigung der individuellen Empfindlichkeit der Ratten in Einzelfällen. - Wegen der bekannten jahreszeitlichen Schwankungen der Empfindlich. keit der Ratte sei erwähnt, daß alle Plasmabestimmungen in

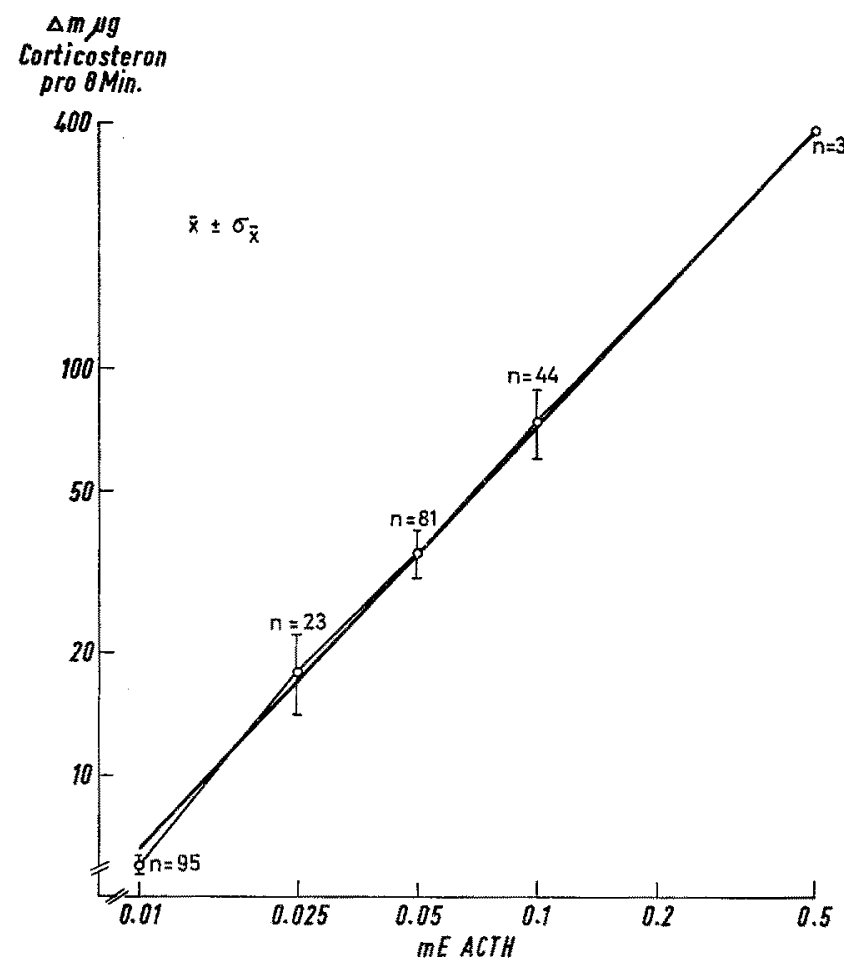

Abb. 2. Bichkurve von Dosis ACTH und Anstieg der Corticasteroninkretion. Angegeben sind die Mittelwerte $(\bar{x})$ und die Standardabweichung der Mittelwerte $\left(\sigma_{\bar{x}}\right)$ von $(n)$ als technisch geglückt anzusehenden Bestimmungen (vgl. Methoden). Uber diese Eichkurve warden die unter Ergebnissen mitgeteiten ACTH-Plasmaspiegel ermittelt

der Zeit von Anfang November bis Ende März durchgeführt wurden. - Die Zahl der Bestimmungen von Plasmawerten ist einerseits durch den großen Zeitaufwand der Methode und andererseits durch die beschränkte Haltbarkeit der ACTHAktivität $[8,10]$ in eingefrorenen $\left(-18^{\circ} \mathrm{C}\right)$ Plasmaproben oder Standardlösungen begrenzt. Alle ACTH-Lösungen und Plasmaproben wurden bei ca. $+2^{0} \mathrm{C}$ möglichst schnell gewonnen, um Aktivitätsverluste zu vermeiden. - Wegen des bekannten Tagesrhythmus der ACTH-Inkretion wurden alle Blutentnahmen bei den Patienten um 6 Uhr morgens begonnen.

\section{Ergebnisse}

Nach den Untersuchungen verschiedener Autoren $[3-6,12,15,21-23,26-32]$ nimmt man heute an, daß der ACTH-Spiegel im Plasma des Normalen zwischen 0,003 und $0,006 \mathrm{mE} / \mathrm{ml}$ liegt. Es war daher nicht besonders überraschend, daß mit der beschriebenen biologischen Bestimmungsmethode normale ACTHSpiegel im Plasma aus der Vena cubitalis nicht zu erfassen waren (Tabelle 1). Aber auch im Plasma aus dem Bulbus cranialis venae jugularis fanden wir keine sicher meBbaren ACTH-Spiegel (Tabelle 1). Die zur Erhaltung des normalen peripheren ACTH-Spiegels erforderliche ACTH-Inkretion des Hypophysenvorderlappens ist demnach offenbar zu gering, um zu einer
Tabelle 1. AOTH.Plasmaspiegel bei 7 Nebennierengesunden

Angegeben sind die Mittelwerte von $(n)$ als technisch gelungen anzusehenden Bestimmungen für jeden einzelnen Patienten. Die Mittelwerte aller Patienten wurden aus den mittleren Corticosteronanstiegen im Nebennierenvenenplasma der Ratte nach Infusion von $1 \mathrm{ml}$ menschlichem Plasma, wie unter Methoden beschrieben, berechnet. Bei diesen Patienten wurden außerdem die sog. 11-Hydroxycorticosteroide im Plasma bestimmt. Der Mittelwert lag mit $29,4 \mu \mathrm{g}-\%$ (6 Uhr morgens) etwas höher als der Normalbereich für $9 \mathrm{Uhr}$ von $5-22 \mu \mathrm{g} * \%$ (18). Eine Stunde nach Injektion von $4 \mathrm{mg}$ Dexamethason war der Mittelwert der sog. 11-Hydroxycorticosteroide auf $16,9 \mu \mathrm{g}$ - \% abgesunken. Der mittlere ACTHPlasmaspiegel war zu diesem Zeitpunkt, um ca. 7.30 Uhr, ebenfalls $<0,01 \mathrm{mE} / \mathrm{ml}(n=18)$. Die Diagnosen sind $\mathrm{Ab}$ schluBdiagnosen.

\begin{tabular}{|c|c|c|}
\hline \multirow[t]{2}{*}{ Patjent } & \multicolumn{2}{|c|}{ mE ACTH pro ml Plasma } \\
\hline & $\begin{array}{l}\text { Bulbus cran. } \\
\text { ven. jugul. }\end{array}$ & $\begin{array}{l}\text { Vena } \\
\text { cubitalis }\end{array}$ \\
\hline $\begin{array}{l}\text { K. M., } 36 \text { Jahre, } \delta \\
\text { Verdacht auf Myopathie }\end{array}$ & $\underset{(2)}{<0,01}$ & $\underset{(2)}{<0,01}$ \\
\hline $\begin{array}{l}\text { B. } 0 ., 47 \text { Jahre, } \delta \\
\text { vegetative Beschwerden }\end{array}$ & $\begin{array}{l}0,015 \\
(1)\end{array}$ & $\begin{array}{l}<0,01 \\
(2)\end{array}$ \\
\hline $\begin{array}{l}\text { R. M., } 20 \text { Jahre, } 0 \\
\text { Zustand nach Schädeltrauma }\end{array}$ & $\begin{array}{l}<0,01 \\
(2)\end{array}$ & $\begin{array}{l}<0,01 \\
(2)\end{array}$ \\
\hline $\begin{array}{l}\text { H. P., } 19 \text { Jahre, } \hat{o} \\
\text { alimentäre Adipositas }(60)\end{array}$ & $\begin{array}{l}0,01 \\
(2)\end{array}$ & $\begin{array}{l}<0,01 \\
(2)\end{array}$ \\
\hline $\begin{array}{l}\text { K. J., } 49 \text { Jahre, } \hat{\delta} \\
\text { Jackson-Anfälle }\end{array}$ & $\begin{array}{l}<0,01 \\
(3)\end{array}$ & $\begin{array}{l}0,01 \\
(2)\end{array}$ \\
\hline $\begin{array}{l}\text { B. J., } 46 \text { Jahre, } 0 \\
\text { traumatische Epilepsie }\end{array}$ & - & $\begin{array}{l}<0,01 \\
(6)\end{array}$ \\
\hline $\begin{array}{l}\text { W. B., } 52 \text { Jahre, } \\
\text { Prostatahypertrophie }\end{array}$ & $\begin{array}{l}<0,01 \\
(3)\end{array}$ & $\begin{array}{l}0,02 \\
(2)\end{array}$ \\
\hline $\begin{array}{l}\text { Mittelwerte } \\
\text { aller Patienten }\end{array}$ & $\begin{array}{l}<0,01 \\
(n=13)\end{array}$ & $\begin{array}{l}<0,01 \\
(n=18)\end{array}$ \\
\hline
\end{tabular}

von uns sicher meBbaren Erhöhung des ACTH-Spiegels im Bulbus cranialis venae jugularis zu führen.

Eindeutig zu erfassen sind dagegen die erhöhten ACTH-Spiegel im Plasma von Patienten mit bestimmten Erkrankungen (Tabelle 2). Beim Morbus Addison sind die ACTH-Werte im Plasma kurz nach Absetzen der Substitutionsbehandlung stark erhöht $[4,12,21$, 33, 34]. Diese ACTH-Spiegel sind weit höher als die z. B. in einer Stress-Situation beim Normalen zu beobachtenden [12]. Das ist zunächst erstaunlich, da alle diese Patienten ja im allgemeinen unter einer als ausreichend anzusehenden Substitutionsbehandlung mit Cortisol stehen. Bedenkt man aber die relativ kurze Halbwertszeit oral applizierter Corticosteroide [35], so kann man sich vorstellen, daß bei einer oralen Substitutionsbehandlung, mit z. B. morgens $20 \mathrm{mg}$ und. nachmittags $10 \mathrm{mg}$ Cortisol, erhebliche Sohwankungen des Plasmacortisolspiegels auftreten. Diese Behandlung kann das feine Spiel der physiologischen Cortisolinkretion der Nebennierenrinde eben nicht vollwertig ersetzen. So wird es auch bei ,korrek $t^{4}$ durchgeführter Substitutionsbehandlung im Laufe der 24 Std eines Tages immer wieder zu Phasen des relativen Cortisolmangels kommen. Die Antwort des Reglers der Cortisolinkretion ist durch seine ständige Überbeanspruchung pathologisch gesteigert. Das sollte der Kliniker im Hinblick auf die bekannten extraadrenalen Wirkungen des ACTH [36] bei der Behandlung von Patienten mit Morbus Addison nicht vergessen.

Der erste Patient der Tabelle 2 (B. S.) litt seit 1952 an einem tuberkulös bedingten Morbus Addison und wurde bis zur Aufnahme im Jahre 1964 mit Desoxycorticosteron behandelt. Intensive Pigmentation und verkalkte Ohrknorpel 
Tabelle 2. ACTH-Plasmaspiegel bei Patienten mit Erkrankungen des ,Hypophysen-Nebennierensystems"

Angegeben sind die Mittelwerte von (n) Bestimmungen für jeden Patienten sowie die Werte der sog. 11-Hydroxycorticosteroide (s. Methoden), Bei den Patienten B. S., G.J., E. L., M. E. und W. L. fand sich um $6 \mathrm{Uhr}$ morgens ein mittlerer ACTH.Plasmaspiegel von $0,206 \mathrm{mE} / \mathrm{ml}(n=13)$ in der Vena cubitalis und von $0,368 \mathrm{mE} / \mathrm{ml}(n=14)$ im Bulbus c. v. j. Das mittlere Körpergewicht dieser 5 Patienten betrug $61,1 \mathrm{~kg}$. - Eine Stunde nach Injektion von $4 \mathrm{mg}$ Dexamethason betrug der mittlere ACTH-Plasmaspiegel im Bulbus c. v. j. $0,086 \mathrm{mE} / \mathrm{ml}(n=12)$, womit er also um etwas mehr als dio Hälfte gegenüber dem Ausgangswert in der V. cubitalis abgesunken war. Nimmt man wie $(30)$ an, daß das Dexamethason die ACTH.Inkretion des Hypophysenvorderlappens sofort und vollständig supprimiert, was nicht ohne weiteres berechtigt erscheint, so paßt diese Abnahme größenordnungsmäßig zo der von uns berechneten Halbwertszeit für endogenes ACTH von ca. $40 \mathrm{~min}$ (s. Diskussion). Die Krankengeschichten der Patienten sind im Text kurz besprochen. Die Patienten mit Nebenniereninsuffizienz erhielten bis $22 \mathrm{Std}$ vor Versuchsbeginn ihre übliche Substitutionsbehandlung.

\begin{tabular}{|c|c|c|c|}
\hline \multirow[t]{2}{*}{ Patient } & \multicolumn{2}{|c|}{ mE ACTH pro ml Plasma } & \multirow{2}{*}{$\begin{array}{l}\text { sog. Plasma. } \\
11-0 \text { HCS } \\
\mu \mathrm{g}-\%\end{array}$} \\
\hline & $\begin{array}{l}\text { Bulbus cran. } \\
\text { ven. jugul. }\end{array}$ & $\begin{array}{l}\text { Vena } \\
\text { cubitalis }\end{array}$ & \\
\hline $\begin{array}{l}\text { B. S., } 48 \text { Jahre, o, } 67,3 \mathrm{~kg} \\
\text { M. Addison }\end{array}$ & $\begin{array}{l}0,45 \\
(4)\end{array}$ & $\begin{array}{l}0,28 \\
(3)\end{array}$ & 4,0 \\
\hline $\begin{array}{l}\text { G. J., } 18 \text { Jahre, } 6,55,5 \mathrm{~kg} \\
\text { M. Addison }\end{array}$ & $\begin{array}{l}0,26 \\
(2)\end{array}$ & $\begin{array}{l}0,24 \\
(2)\end{array}$ & $\underline{7,0}$ \\
\hline $\begin{array}{l}\text { E. L., } 19 \text { Jahre, } 0,50,5 \mathrm{~kg} \\
\text { Zustand nach totaler } \\
\text { Adrenalektomie }\end{array}$ & $\begin{array}{l}0,27 \\
(4)\end{array}$ & $\begin{array}{l}0,04 \\
(3)\end{array}$ & 5,0 \\
\hline $\begin{array}{l}\text { M. E., } 44 \text { Jahre, }, 9,82,0 \mathrm{~kg} \\
\text { Zustand nach totaler } \\
\text { Adrenalektomie }\end{array}$ & $\begin{array}{l}0,06 \\
(2)\end{array}$ & $\begin{array}{l}0,03 \\
(2)\end{array}$ & $(10,0 ?)$ \\
\hline $\begin{array}{l}\text { W.L., } 21 \text { Jahre, } 9,50,0 \mathrm{~kg} \\
\text { Adrenogenitales } \\
\text { Syndrom }\end{array}$ & $\begin{array}{l}0,80 \\
(2)\end{array}$ & $\begin{array}{l}0,44 \\
(3)\end{array}$ & $, 22,0^{46}$ \\
\hline $\begin{array}{l}\text { L. J., } 61 \text { Jahre, } \hat{} \\
\text { Bronchial-Ca, HVL- } \\
\text { Metastasen, sek. NNR- } \\
\text { Insuffizienz }\end{array}$ & $\underset{(4)}{<0,01}$ & $\begin{array}{l}<0,01 \\
(3)\end{array}$ & 3,0 \\
\hline
\end{tabular}

(Thorns Zeichen, [61], sprachen für eine nicht ganz ausreichende Substitution der Nebennierenrindeninsuffizienz. Wir fanden sehr hohe ACTH-Spiegel in der Vena cubitalis. Der ACTH-Spiegel im Bulbus cranialis venae jugularis war noch höher. - Der zweite Patient mit einem Morbus Addison (G.J.) erkrankte Ende 1964 mit einer Nebennierenrindeninsuffizienz, deren erstes Zeichen ein deutliches Nachlassen der Konzentrations- und Merkfähigkeit war [37].

Im Jahre 1958 beschrieben Neuson u. Mitarb. den ersten Fall, bei dem man die Entwicklung eines chromophoben Adenoms des Hypophysenvorderlappens nach totaler Adrenalektomie wegen eines Cushing. Syndroms mit bilateraler NNR-Hyperplasie beobachtete und exzessiv hohe ACTH-Spiegel im Plasma fand [38]. Seither wurden wiederholt Beobachtungen solcher Fälle mitgeteilt (z.B. [39, 40]). Die gesteigerte ACTH-Inkretion bei diesen adrenalektomierten Patienten ist nicht ohne weiteres mit der von AddisonKranken vergleichbar, da unseres Wissens bei letzteren die Entstehung von Hypophysenvorderlappenadenomen nich t beobachtet wurde [41]. Aus Tierexperimenten [42] kennt man allerdings das Wachstum von ACTH. produzierenden HVL-Adenomen nach Entfernung der Nebennieren.

Ein 19jähriger Mann (E. L.) ist seit dem 15. Lebensjahr in unserer Beobachtung. Er wurde seinerzeit wegen eines typischen Cushing-Syndroms mit bilateraler Hyperplasie der Nebennierenrinde totaladrenalektomiert. Das führte za einer vollständigen Remission des Krankheitsbildes und zu einer
Umkehr der vorher latent diabetischen Stoffwechsellage in eine erhöhte Glucosetoleranz [37. 43]. Jetzt findet sich bei dem Patienten eine intensive Pigmentation und ein doppelt konturierter Sellaboden. Nach der Tomographie ist die Sella einseitig vergrößert, so daß wir den Patienten unter dem Verdacht auf das Vorliegen eines sog. chromophoben Adenoms bzw. R-Zellen-Adenoms [44] weiter beobachten. Wie aus Tabelle 2 ersichtlich, waren die ACTH-Spiegel bei diesem Patienten sehr hoch.

Auch beim adrenogenitalen Syndrom, bei dem es sich bekanntlich in typischen Fällen zumeist um eine unvollständige Blockierung der 21 - Hydroxylierung [45] handelt, was zu einer verminderten Cortisolproduktion, einer vermehrten ACTH-Aussehüttung und einer erhöhten Androgenproduktion in der Nebenniere führt, kann man erhöte ACTH-Spiegel messen [12]. Eine 23jährige Patientin (W. F.), bei der die Diagnose schon vor Jahren gestellt worden war, infolge ihrer Indolenz jedoch keine Behandlung erfolgte, hatte extrem hohe ACTH-Spiegel im Bulbus cranialis venae jugularis und etwa um die Hälfte niedrigere Spiegel in der Vena cubitalis.

Klinisch interessante Gesichtspunkte bot die Krankengeschichte des Patienten L. J. (Tabelle 2). Dieser Patient wurde wegen eines Bronchialcarcinoms (Plattenepithelcarcinom) beobachtet. Adynamie, ausgeprägte Hypotonie, Erbrechen und Hyponatriämie ließen eine Nebennierenrindeninsuffizienz annehmen. Die „Plasmacortisolwerte" waren erniedrigt. Überraschenderweise fanden sich weder im Bulbus cranialis venae jugularis noch in der Vena cubitalis meßbare ACTH-Plasmaspiegel, die bei den Addison-Patienten deutlich erhöht waren. Bei der Autopsie ergab sich die Erklärung dieses Ergebnisses. Der Hypophysenvorderlappen war durch Metastasen des Bronchialcarcinoms vollständig zerstört, während die Nebennieren nur kleine Metastasen aufwiesen. Es handelte sich somit um eine sekundäre Nebennierenrindeninsuffizienz. Während der klinischen Beobachtung war daran auch schon gedacht worden, da dieser Patient mit einer ausgeprägten Kälteemptindlichkeit und Bradykardie sowie einer Verlangsamung der Achillessehnenreflexe die Zeichen einer sekundären Hypothyreose aufwies.

\section{Diskussion}

Die gesamte im Körper zu einem beliebigen Zeitpunkt befindliche Menge an ACTH berechnet sich durch Multiplikation des fiktiven Verteilungsvolumens für ACTH mit dem ACTH-Spiegel in der Peripherie (Vena cubitalis). Durch Multiplikation mit 0,5 erhält man die Menge an ACTH, die in einer Halbwertszeit für ACTH eliminiert bzw. von der Hypophyse produziert wird (Zähler von Gleichung 1). Diese ACTHMenge wird im Gleichgewicht pro Halbwertszeit von der Hypophyse ersetzt und verteilt sich zunächst aut diejenige Plasmamenge, die in einer Halbwertszeit durch das Hirnkreislaufgebiet fließt. Diese Plasmamenge kann im Bulbus cranialis venae jugularis erfaßt werden und läßt sich aus Hirndurchblutungsvolumen pro Minute, Hämatokrit und Halbwertszeit für endogenes $\mathrm{ACTH}$ berechnen:

\begin{tabular}{|c|c|c|c|c|c|c|}
\hline \multicolumn{7}{|c|}{ Gleichung 1} \\
\hline \multirow{3}{*}{$\triangle \mathrm{ACTH}=$} & $0,5 \times$ & $\begin{array}{l}\text { Verteilungs- } \\
\text { volumen }\end{array}$ & $7 x$ & $\mid \begin{array}{l}\text { ACTH } \\
\text { Plasma- }\end{array}$ & \multirow{2}{*}{$1 \times$} & $\mathrm{mE}$ \\
\hline & & ACTH & & spiegel & & I \\
\hline & $\begin{array}{l}\mathrm{Ce} \\
\mathrm{Pl} \\
\mathrm{prc}\end{array}$ & $\left.\begin{array}{l}\text { rebraler } \\
\text { manafiub } \\
\text { Minute }\end{array}\right] \times$ & $\begin{array}{l}\text { Hal } \\
\text { zeit } \\
\text { ACl }\end{array}$ & $\begin{array}{l}\text { bwerts- } \\
\text { rH }\end{array}$ & $\frac{1}{\min }$ & $\min$ \\
\hline
\end{tabular}

Dabei ist: $\triangle \mathrm{ACTH}=$ Zunahme des ACTH-Spiegels im Hirnkreislauf in $\mathrm{mE} /$ Liter. Verteilungsvolumen für ACTH $=$ $43 \%$ des Körpergewichtes nach WoLF u. Mitarb. [46] für ${ }_{131}$ Jod- $\beta^{1-24}$-Corticotrophin. Bei einem mittleren Körpergewicht von $61,1 \mathrm{~kg}$ der fünf Patienten mit erhöhten ACTH-Spiegeln (Tabelle 2) ist ihr mittleres Verteilungsvolumen für ACTH also 26,3 Liter. 
Cerebraler Plasmafluß pro Minute $=$

Hirndurchblutung $(0,8$ Liter/min nach $[1,2]) \times$

$$
\times\left[\frac{100-\text { Hämatokrit }}{100}\right]=\frac{0,8 \cdot 55}{100}\left(\frac{1}{\min }\right)
$$

Folgende Voraussetzungen haben zu gelten, damit die angegebene Gleichung gültig sein kann. Der ACTH-Spiegel im Plasma aus der Vena cubitalis muß repräsentativ fứr das gesamte Verteilungsvolumen sein. Diese Annahme ist ja Voraussetzung für die Berechnung des fiktiven Verteilungsvolumens durch retrograde Extrapolation aus dem Abfall der Plasmakonzentration des ${ }^{131}$ Jod-ACTH [46]. Das fiktive Verteilungsvolumen ist eine rechnerische Größe, die anatomisch nicht ohne weiteres zu definieren ist. Für Parathormon wurde ein ähnliches Verteilungsvolumen, nämlich $36,4 \%$ des Körpergewichtes [47], angegeben. Weiter muß man voraus. setzen, daB alles ACTH den Schädelinnenraum über die Bulbi craniales venae jugularis verlä $B t[48,49]$ und sich in der kurzen Zeit bis zur Passage des Bulbus cranialis venae jugularis ledig. lich im Plasma and nicht z.B. auf die Erythrocyten verteilt. Sollte ein ins Gewicht fallender Teil des venösen Blutes aus dem Hypophysenvorderlappen auf anderen Wegen (z.B. Rete venosum foram. oval.) das Schädelinnere verlassen, so würden sich zu lange Halbwertszeiten für endogenes ACTH berechnen. Weitere Voraussetzungen sind, daß der Verteilungsraum von endogenem ACTH gleich dem von ${ }^{131}$ Jod-ACTH ist, und die Plasmraspiegel im venösen Blut nach Passage der terminalen Hirnstrombahn nicht höher oder niedriger als $z . B$, in der Vena cubitalis sind.

Es ist klar, daß nicht alle diese Voraussetzungen ohne weiteres zu akzeptieren sind. Berechnet man trotzdem aus den Mittelwerten der gemessenen ACTHSpiegel in der Vena cubitalis (206 $\mathrm{mE} /$ Liter) und der mittleren Erhöhung des ACTH-Spiegels im Bulbus cranialis venae jugularis (162 mE/Liter) nach Gleichung 1, in der das Verteilungsvolumen und der cerebrale Plasmafluß bei bekanntem Körpergewicht konstante Größen sind und die einzige Unbekannte die Halbwertszeit für ACTH ist, diese Halbwertszeit für endogenes ACTH, so ergibt sich - bei Berücksichtigung der fünf Patienten (B.S., G.J., E. L., M. E. und W.F., Tabelle 2) - eine Halbwertszeit von $38 \mathrm{~min}$. In Anbetracht der nicht unerheblichen Voraussetzungen and der relativ kleinen Zahl der untersuchten Patienten möchten wir diese Halbwertszeit lediglich als größenordnungsmäßige Abschätzung und nicht als genaue Bestimmung verstanden wissen.

Die in der Literatur angegebenen Werte für die Halbwertszeit von exogenem, markiertem oder nicht markiertem, also biologisch gemessenem ACTH sind zum Teil erheblich kürzer $[4,30,46,47,50-59]$. Allerdings fanden Pfeirfer u. Mitarb. [4] bei Infusion von $25 \mathrm{E}$ artfremdem ACTH über 4 Std unter Verwendung einer biologischen Bestimmungsmethode, welche der unseren ähnlich ist, eine Halbwertszeit von 29 min und berichteten ferner, daß die Halbwertszeit von schnell injiziertem ACTH kürzer und dosisabhängig sei. Nexson u. Mitarb. [34, 51] fanden ebenfalls eine Halbwertszeit für Schweine-ACTH, die etwas länger als nur wenige Minuten war.

Bei Patienten, die nach den klinischen Befunden keine Störungen der Nebennierenrindenfunktion aufwiesen, fanden wir weder in der Vena cubitalis noch im Bulbus cranialis venae jugularis meßbare ACTHSpiegel. Nimmt man willkürlich einen normalen ACTH-Spiegel von $6 \mathrm{mE} /$ Liter Plasma an und berücksichtigt, da.B die Empfindlichkeitsgrenze der Bestimmungsmethode bei etwa $0,01 \mathrm{mE}$ (obere Grenze des $95 \%$ Vertrauensbereiches $=0,05 \mathrm{mE}$, s. Methoden) liegt, so läBt sich nach Umformung von Gleichung 1 errechnen, daB die Halbwertszeit für endogenes ACTH kleiner als 4 min sein müBte, damit die Spiegel im Bulbus cranialis venae jugularis signifikant höher als in der Vena cubitalis sein könnten. Dieser Berechnung, aus der umgekehrt geschlossen werden kann, daß die biologische Halbwertszeit von endogenem ACTH größer als $4 \mathrm{~min}$ sein muß, wurde ebenfalls ein Verteilungsvolumen für ACTH von 26,3 Litern zugrunde gelegt.

Es ist von anderen Autoren versucht worden, mit etwas anderen Methoden den Normalspiegel von ACTH zu bestimmen. Die Arbeitsgruppe von LIDDLE hat ein Verfahren angegeben, ACTH aus größeren Plasmamengen anzureichern und mit einer biologischen Methode zu bestimmen [12, 22, 27]. Auch andere Autoren haben diese Möglichkeit untersucht [21, 28]. Die Ausbeuten liegen bei diesem Anreicherungsverfahren zwischen 44 und $124 \%$. Zu diesem Fehler addiert sich der Fehler der biologischen Bestimmungsmethoden. Immerhin verdankt man diesen Untersuchungen die Vorstellung, dal der normale Plasmaspiegel an ACTH zwischen 3 und $6 \mathrm{mE} \mathrm{je}$ Liter Plasma liegt. - Einen ganz anderen Weg gingen Untersucher, die sich des Prinzips der radioimmunologischen Bestimmung von Peptidhormonen für die ACTH-Bestimmung bedienten [30-32]. Diese Autoren berichteten über eine ganz ausgezeichnete Empfindlichkeit dieses Verfahrens $(0,1 \mu \mathrm{E})$. Es wurden zunächst allerdings um drei Zehnerpotenzen höhere Normalspiegel gefunden, als sie bei den biologischen Bestimmungsmethoden gesehätzt wurden [31]. Ein weiterer Einwand gegen dieses Verfahren lkommt aus der Arbeitsgruppe von Forsmam. [32]. Ein synthetisches Peptid aus den Aminosäuren Nr. 22-39 der Sequenz des vollständigen $\beta^{1-39}$ Corticotrophins zeigte die volle immunologische Aktjvität, ohne daß eine biologische Aktivität nachweisbar war.

Bei den fünf Patienten mit erhöhten ACTHSpiegeln (Tabelle 2) wurde ein mittlerer ACTHPlasmaspiegel von $0,206 \mathrm{mE}$ ACTH/ml Plasma in der Vena cubitalis gefunden. Im Plasma aus dem Bulbus cranialis venae jugularis waren die ACTH-Spiegel im Mittel um 0,162 mE ACTH/ml höher (Signifikanz der mittleren Differenz: $p<0,05$ ). Unter der Annahme einer Hirndurchblutung von $0,8 \mathrm{Liter} / \mathrm{min}[1,2]$ läBt sich für diese Patienten, ohne Berücksichtigung des Tagesrhythmus, eine ACTH-Inkretion von ca. $100 \mathrm{E}$ je Tag errechnen. Die naturgemäß wesentlich niedrigere ACTH-Inkretionsrate bei normalen ACTH-Spiegeln ist aus unseren Daten nicht zu berechnen.

Es ist zu hoffen, daß Verbesserungen der Methoden der ACTH-Bestimmung in Zukunft die Kenntnis um die dynamischen Verhältnisse der ACTH-Inkretion erweitern werden.

Die Autoren danken Frau D. SEIBERT und Frl. F. SEDLMAIER für ihre sorgfältige technische Assistenz. Wesentliche Teile dieser Arbeit werden von R. HACKER und F. KLUGE der Medizinischen Fakultät der Universität München in ihren Dissertationsarbeiten vorgelegt.

Zusammenfassung. Der Anstieg der Corticosteroninkretion in das Nebennierenvenenblut frisch hypophysektomierter Ratten diente zur Bestimmung von ACTH-Spiegeln in $1 \mathrm{ml}$ nativen, menschlichen Plasma. Normale ACTH-Plasmaspiegel sind sowohl bei Punktion der Vena cubitalis als auch des Bulbus cranialis venae jugularis durch diese Methode nicht oder nur ungenau zu erfassen. Bei Patienten mit pathologisch erhöhten ACTH-Spiegeln in der Vena cubitalis sind die ACTH-Spiegel im Bulbus cranialis venae jugularis signifikant höher. Es ließ sich eine Beziehung zwischen ACTH-Spiegel in der Peripherie (Vena cubitalis), Differenz der ACTH-Spiegel zwischen Bulbus cranialis venae jugularis und Vena cubitalis und biologischer Halbwertszeit von endogenem ACTH aufstellen. Nach den Frgebnissen der Bestimmung von ACTH-Spiegeln bei Nebennierengesunden läßt sich folgern, daß die 
biologische Halbwertszeit von endogenem ACTH größer als 4 min sein muß. Bei Patienten mit erhöhten ACTH-Spiegeln ließ sich die biologische Halbwertszeit von endogenem ACTH größenordnungsmäßig mit ca. $40 \mathrm{~min}$ berechnen. Bei diesen Patienten betrug die mittlere tägliche ACTH-Inkretion ca. $100 \mathrm{E}$.

Summary. ACTH-contents of $1 \mathrm{ml}$ specimens of human plasma were assayed by measurement of increases of corticosterone output in the adrenal vein of acutely hypophysectomized rats. This procedure is not sensitive enough to measure normal ACTH-levels acurately, neither when blood was drawn from the bulbus cranialis venae jugularis, nor from the vena cubitalis. In patients having pathologically elevated ACTH-levels, the ACTH-content of plasma is significantly higher in the bulbus cranialis venae jugularis than in peripheral venous blood. An equation is presented formulating the relation of peripheral ACTHlevels, differences of ACTH-levels between bulbus cranialis venae jugularis and vena cubitalis, and of the biological halflife of endogenous ACIH. On the basis of the results of the determinations of socalled normal ACTH-levels it can be concluded, that the biological halflife of endogenous ACTH is longer than $4 \mathrm{~min}$. From the data of patients with elevated ACTHlevels a halflife of approximately $40 \mathrm{~min}$ and a mean ACTH-secretion of approx. 100 units per day could be calculated.

Literatur. [1] KeTY, S. S., and C. F. Schmint: The nitrous oxide method for the quantitative determination of cerebral blood flow in man: Theory, procedure and normal values. J. clin. Invest. 27, 476 (1948). - [2] BerssMEIER, A., U. GotTSTEIN u. W. RUDoLPH: Herzkrankheiten als Ursache cerebraler Zirkulationsstörungen. Dtsch. med. Wschr. 87, 16 (1962). - [3] Lrrscoms, H. S., and D.H. NELson: A sensitive biologic assay for ACTH. Endocrinology 71, 13 (1962). - [4] Pfelffer, E. F., F. Garmendia, E. VAUBeL u. K. RETLENE: Exogene und endogene ACTHAktivitäten im nativen Plasma des Menschen. Ergebn. inn. Med. Kinderheilk. N. F. 20, 127 (1963). - [5] GIRARD, F., and V.K. VANCE: Modification of the Lipscomb-Nelson bioassay for ACTH. Endocrinology 71, 325 (1962). - $[6]$ RETTENE, K., H. Ditschuneit, M. Fischer, K. Kopp u. E. F. Pfeiffer. Corticotropin-Bestimmung anhand des Corticosteron-Anstieges in Nebennierenvenenblut hypophysektomierter Ratten. Vergleich von Dexamethasonblockade und Hypophysektomie. Acta endocr. (Kbh.) 41, 24 (1962). - [7] Frevd, J.: Die Hypophysektomie. In: Handbuch der biologischen Arbeitsmethoden. Hrsg. E. Abderhald we, Abt. V, Teil 3, B II, S. 1442. Berlin: Urban \& Schwarzenberg 1938. - [8] HACKER, R.: Diss. Universität München, 1966. - [9] KuvGE, H.: Diss. Universität München, 1967 (in Vorbereitung). - [10] ScRTBA, P. C.: Habilitationsschrift Universität München, 1966. [11] Harrison, R. G., and M. J. HoEy: The adrenal circulation. Oxford: Blackwell Sci. Publ. 1960. - [12] Lroole, G. W., D. IsLAND, and C. K. Mrador: Normal and abnormal regulation of corticotropin secretion in man. Recent Progr. Hormone Res. 18, 125 (1962). - [13] OHSAWA, N.: Effect of administration of ACTH on adrenal responsiveness of hypophysectomized rats. Endocrinology 77, 461 (1965). - [14] Z kiNKER, N., and D. E. Bernstern: The estimation of small amounts of corticosterone in rat plasma. J. biol. Chem. 281, 695 (1958). - [15] Pferffer, E. F., W. E. VAUbeL, K. RetreNE, D. Berg u. H. DTtschuneIT: ACTH-Bestimmung mittels Messung des Plasma-Corticosterons der mit Dexamethason hypophysenblockierten Ratte. Klin. Wschr. 38, 980 (1960). [16] DE Moor, P., and O. Streexo: Comparison of three techniques for the fluorimetric determination of plasma corticosteroids. J. Endocr. 28, 59 (1963). - - 177] MATTINGLY, D., P. M. Dennis, J. Pearson, and C. L. Cope: A simple fluorimetric method for the estimation of free 11-hydroxycorticoids in human urine. Lancet 1964 II, 1046. - [18] HochHeUs ER, W., M. Müller-BardorfF, P. C. Scriba u. K. Schwarz:
Fluorimetrische Bestimmung der 11-Hydroxycorticosteroide im Plasma unter der Therapie mit Corticoiden. 12. Sympos. Dtsch. Ges. Endokrinol., Wiesbaden. Berlin-Heidelberg-New York: Springer 1966 (im Druck). - [19] URQUHART, J.: Adrenal blood flow and the adrenocortical response to corticotropin. Amer. J. Physiol. 209, 1162 (1965). - [20] PorTer, J. C., and M. S. KLAIBER: Corticosterone secretion in rats as a function of ACTH input and adrenal blood flow. Amer. J. Physiol. 209, 811 (1965). - [21] VANCE, V. K., W. J. ReDDY, D. H. NeLson, and G. W. THorN: ACTH in human plasma. J. clin. Invest. 41, 20 (1962). - [22] NEY, R. L., N. SHIMtzd, W. E. NICHoLSON, D. P. IstaAND, and G. W. LIDDde: Correla. tion of plasma ACTH concentration with adrenocortica] response in normal human subjects, surgical patients, and patients with Cushing's disease. J. clin. Invest. 42, 1669 (1963). - [23] Retrene, K., A. Espinoza, K. H. MarX u. E. F. PFitfere: Uber das Verhalten von ACTH und Cortisol im Blut von Normalen und von Kranken mit primärer und sekundärer Störung der Nebennierenrindenfunktion. Klin. Wschr. 43, 205 (1965). - [24] DrEM, K.: Documenta Geigy, Wissenschaftliche Tabellen, 6. Aufl. Basel: J. R. Geigy S. A. 1960. - [25] GADDUN, J. H.: Bioassay and mathematics. Pharmacol. Rev. 5, 87 (1953). - [26] SrDnor, K. L., and G. SAYERS: Blood and pituitary ACTH in intact and adrenalectomized rats after stress. Endocrinology 55, 621 (1954). [27] Wrultams, W. L., D. Istand, R. A. A. Oldftetd, and G. W. LrDDLe: Blood corticotropin (ACTH) levels in Cushing's disease. J. olin. Endocr. 21, 426 (1961). - [28] Davres, B. M. A.: Blood corticotrophin in normal adults and in patients with Cushing's syndrome. Acta endocr. (Kbh.) 45, 55 (1964). - [29] Porves, H. D., and N. E. Siretr: Assay of corticotrophin in dexamethasone-treated rats. Endocrinology 77, 366 (1965). - [30] YaLOW, R. S., S. M. GLroK, J. RoTH, and S.A. Berson: Radioimmunoassay of human plasma ACTH. J. clin. Endocr. 24, 1219 (1964). - [31] FELBerR, J. P., A. J. Moody, A. Villanueva et A. Vanotiti: Determination radio-immunologique de l'insuline et de l'ACTH en clinique. Bull. sehweiz. Akad. med. Wiss. 21, 261 (1965). [32] IMUra, H., L. L. Sparks, G. M. Grodsky, and P. H. ForshaM: Immunologic studies of adrenocorticotropic hormone (ACTH). Dissociation of biologic and immunologic activities. J. clin. Endocr. 25, 1361 (1965). - [33] GRABRR, A. L., J. R. GIVENS, W. E. Nicholson, D. P. Istand, and G. W. LIDDLE: Persistence of diurnal rhythmicity in plasma ACTH concentrations in cortisol-deficient patients. J. clin. Endocr. 25, 804 (1965). - [34] NarsoN, D. H. Corticotropin in human plasma: Clinical endocrinology, vol. I, p. 626. New York: Grune \& Stratton, Inc. 1960. - [35] RAITH, L., u. H. J. Kard: Biologische Halbwertszeit, Abbau und Ausscheidung von 16-Methylenprednisolon. Klin. Wschr. 44, 298 (1966). - [36] ENGEL, F. L.: Extraadrenal actions of adrenocorticotropin. In: Vitamins and hormones, p. 194. New York: Academie Press 1961. - [37] Schwarz, K., u. P. C. Scrira: Endokrin bedingte Encephalopathien. Ref. Verh. dtsch. Ges. inn. Med. 72 (1966) (im Druck) - [38] NaLsoN, D. H., J. W. Meaktn, J. B. Dealy, D. D. Matson, K. Emerson, and G. W. THORN: ACTH-producing tumor of the pituitary gland. New Engl. J. Med. 259, 161 (1958). - [39] NeLson, D. H., J. W. MEAKIN, and G. W. THORN: ACTH-producing pituitary tumors following adrenalectomy for Cushing's syndrome. Ann. intern. Med. 52,560 (1960). - [40] MaraUTH, F.: Differentialdiagnostik der Geschwülste im Bereich des Türkensattels. Dtsch. med. Wschr. 89, 1839 (1964). — [4l] KracHr, J.: Das Hypophysennebennierenrinden-System bei Cushing-Syndrom. Verh. dtsch. Ges. Path. 47, 280 (1963). - [42] Taknmoto, H., K. Yororo, J. FormH, and A. T. CoHEn: Adrenotropic acitivity of mammo-somatotropic tumors in rats and mice. Cancer Res. 22, 917 (1962). - [43] SorrwaRz, K., P. C. Sortba u. G. G. Hofmanta: Die intravenöse GlucoseBelastung und die Erfassung einer latenten Nebennierenrindeninsuffizienz während und nach Corticosteroidtherapie. Verh. Dtsch. Ges. inm. Med. 71, 360 (1965). - [44] Kracht, J., H. D. ZTMMERMANN u. U. HaChMETSTER: Immunhistologischer ACTH-Nachweis in einem R-Zellen-Adenom des Hypophysenvorderlappens bei M. Cushing. Virehows Arch. path. Anat. 340, 270 (1966). - [45] BARTTER, F. C., F. Albright, A. P. Forbes, A. Liat, E. Denpsey, and E. CARROLL: The effects of adrenocorticotropic hormone and cortisone in the adrenogenital syndrome associated with congenital adrenal hyperplasia: an attempt to explain and correct its discordered hormonal pattern. J. clin. Invest. 30, 237 (1951). [46] Wolf, R. L., M. Mendlowitz, L.J. Soffers, J. Roboz, 
and S. E. GLtow: Metabolism of corticotropin in man. Proc. Soc. exp. Biol. (N. Y.) 119, 244 (1965). - [47] MELICK, R.A., G. D. AURBaCh, and J.T. Potrs: Distribution and half-life of 131-I-labeled parathyroid hormone in the rat. Endocrinology 77, 198 (1965). - [48] HARRIS, G. W.: Entwicklung und heutiger Stand der Neuroendokrinologie. Dtsch. med. Wschr. 90, 61 (1965). - [49] ANDERson, E.: The hypothalamus and adrenocorticotrophic hormone release. In: The adrenal cortex (ed. H. D. MooN), p. 189. New York: P. B. HoEBer Inc. 1961. - [50] Braunsterner, H., G. GIEBISCH, H. Kolder u. G. Werner: Untersuchungen über die Freisetzung and Verteilung von adrenocorticotropem Hormon in Parabiosetieren. Naunyn-Schmiedebergs Arch. exp. Path. Pharmak. 215, 210 (1952). - [51] MEakn, J. W., J. E. Bethune, R. H. Despolntes $u$. D. H. Nelson: The rate of disappearance of ACTH activity from the blood of humans. J. elin. Endocr, 19, 1491 (1959). - [52] MEAKIN, J. W., W. H. TINGEY, and D. H. NeLson: The catabolism of ACTH: Stability in blood, plasma, serum, saline. Endocrinology 66, 59 (1960). - [53] Meamon, J. W., and D. H. NeLson: The catabolism of AOTH: Some characteristics of an ACTH inactivating system in plasma. Endocrinology 66, 73 (1960). [54] RrCHards, J. B., and G. SAynrs: Fate and excretion of adrenocorticotropic hormone. Proc. Soc. exp. Biol. (N. Y.) 77, 87 (1951). - [55] van Drke, D. C., M. E. Stmpson, C. H.
LI, and S. M. Evars: Survival in the circulation of the growth and adrenocorticotropic hormone as evidenced by parabiosis. Amer. J. Physiol. 163, 297 (1950). - [56] GREENSPAN, F. S., C. H. LI, and H. M. Evans: Disappearance rate of ACTH from rat's plasma after i.v. injection. Endocrinology 46, $261(1950)$. - [57] SYDnor, K. L., and G. SAYERS: Biological half-life of endogenous ACTH. Proc. Soc. exp. Biol. (N. Y.) 83, 729 (1953). - [58] Retitene, K., M. Frscher, H. Ditschuneit u. E. F. Preitrater: Biologische Halbwertszeit von exogenem ACTH im menschlichen Blut. 9. Symp. Dtsch. Ges. Endokrinol. Berlin-Göttingen-Heidelberg: Springer 1962. - [59] Porter, J. C., and M. S. KLAIBER: Relationship of imput of ACTH to secretion of corticosterone in rats. Amer. J. Physiol. 207, 789 (1964). - [60] SORGE, F., H. E. Mentzel, G. Schumany, A. Brendel u. E. F. PfeifFER: Sekretionsrhythmik von ACTH und Cortisol im Blute von Stoffwechselgesunden, Prädiabetikern und Fettsüchtigen. Diabetiologia 1, 151 (1965). - [61] THoRn, G. W.: The diagnosis and treatment of adrenal insufficiency, 2 nd ed. Springfiedl (III.): Ch. C. Thomas 1951 Publ.

Dr. med. Perter C. Scriba

Priv.-Doz. Dr. med. K. Schwarz II. Medizinische Universitätsklinik 8 München 15, Ziemssenstr. 1

\section{Immunologische Darstellung zweier verschiedener Anti- $\gamma$-Globulin-Faktoren (Rheumafaktoren)}

\section{H. Mathues, I. ImRe und H. Gaedicke}

Rheumatiker-Ambulanz der Medizinischen Poliklinik der Universität München (Direktor: Prof. Dr. W. SErTz)

Immunologische Untersuchungen des Rheumafaktors setzen seine Isolierung und die Gewinnung eines spezifischen Antiserums voraus, um die gefundenen Präcipitationslinien determinieren zu können. Die Isolierung der Rheumafaktoren aus verschiedenen Polyarthritisseren wurde nach einer von uns bereits beschriebenen Methodik $[4,5,2]$ durchgeführt. Nach Reaktion des Rheumafaktors im Polyarthritisserum mit $\gamma$-Globulin-beladenen Latexpartikeln wurde zentrifugiert und der Rückstand mehrfach gewaschen. Nach Auffüllen mit Pufferlösung erfolgte dann die $\mathrm{Ab}$ sprengung des Rheumafaktors durch Erhitzen auf $63-65^{\circ} \mathrm{C}$. Nach Abzentrifugieren der Latexpartikel bei gleicher Temperatur verbleibt der nach Abkühlen wieder reaktionsfähige Rheumafaktor im Überstand. Im noch in der Wärme abgehobenen Überstand liegt also der Rheumafaktor isoliert vor und kann durch Dialyse weiter konzentriert werden.

Wir haben den Rheumafaktor aus verschiedenen Polyarthritisseren isoliert und durch Immunisierung von Kaninchen Antiseren gewonnen [5]. Mit den Rheumafaktoren und spezifischen Antiseren wurden dann immunologische Untersuchungen mit der Doppeldiffusionsmethode nach OtCHTERLONY und mit der Immunoelektrophorese durchgeführt.

\section{Untersuchungen}

Materialien, Reagentien und apparative Ausrïstung. a) Rheumafaktorpositive (RA-Test), hochtitrige Polyarthritisseren. b) Isolierte Rheumafaktoren (Isolierung nach früher mitm geteilter Methodik $[2,4,5]$.

c) Antirheumafaktorseren (Gewinnung nach früher mitgeteilter Methodik [5].

d) Antihumanserum (Vollserum) von Ziegen (Hyland Laboratories).

e) Anti- $\beta_{2}$-Makroglobulinserum (neuere Nomenklatur: Anti- $\gamma M_{-}$-Globulinserum) von Ziegen (Hyland Laboratories).

f) Anti-7 S- $\gamma$-Globulinserum (neuere Nomenklatur: Anti$\gamma_{G^{-}}{ }^{-G l o b u l i n s e r u m)}$ von Ziegen (Hyland Laboratories). g) Anti-Cohn-Fraktion II-( $\gamma$-Globulin-)Serum von Kaninchen (Hyland Laboratories).

h) Reinagar (Behring-Werke).

i) Phosphatpufferlösung 0,15 molar pH 7,1 zur Herstellung des Agargels für Doppeldiffusionstest.

k) Cialit (Asid-Institut) zur Beigabe zum Agar als Bacteriound Mycostaticum.

1) Veronalpuffer $\mathrm{pH} \mathrm{8,6}$ zur Herstellung des Agargels für Immunoelektrophorese.

m) Veronalpuffer pH 8,6 als Brückenpuffer.

n) Amidoschwarzlösung zur Färbung der Präparate.

o) Acetatpuffer für Amidoschwarzlösung.

p) $2 \%$ ige Essigsäure (Entfärber).

q) Immunoelektrophoresegerät Agafor 1 (Egaton A.G.).

Methodiken. 1. Doppeldiffusion in Agargel, modifiziert nach Ouohterzony [7]: Beschicken von in Petrischalen liegenden Glasplatten (Diagläser $50 \times 50 \mathrm{~mm}$ ) mit $0,8 \%$ igem Agar. Einstanzen von Antigen- bzw. Antikörperlager von $8 \mathrm{~mm}$ Durchmesser im Abstand von ebenfalls $8 \mathrm{~mm}$ und Einfüllen der be. treffenden Seren. Lagerung der mit Deckel verschlossenen Präparate für 48 Std bei Zimmertemperatur. Umschneiden der Agarschicht am Rande der Glasplatten und Entnahme der Platten mit der darauf befindlichen Agarschicht, Waschen (24-48 Std), Färben (10-20 min) und Eintfärben.

2. Immunoelektrophorese, Mikromethodik, gering modifiziert nach SCHEIDEGGER [8]: Beschicken von Objektträgern $(26 \times 76 \mathrm{~mm})$ mit 1,2\%igem Agar und Ausstanzen der betreffenden Antigen- und Antikörperlager mit speziell angefertigten Stanzen. Einfüllen der zu untersuchenden Antigene und Einlegen in die Immunoelektrophoresekammer, Auftrennungszeit $45 \mathrm{~min}$ bei $6 \mathrm{Volt} / \mathrm{cm}$. Nach Herausnahme Einfüllen der Antiseren in die Antikörperlager und Lagerung in feuchter Kammer für 16-18 Std bei Zimmertemperatur. Waschen (24 Std), Trocknen (im Brutschrank bei $37^{\circ} \mathrm{C}$ ), Färben (10-20 min) und Entfärben.

\section{Ergebnisse und Diskussion}

$A b b .1$ demonstriert zunächst ein Rheumatikerserum, das in der Ouchterlony-Platte gegen drei verschiedene Antirheumafaktorseren eine sehr starke Präcipitationslinie mit $\beta_{2}$-Makroglobulincharakter zeigt. Das Rheumatikerserum ergibt gegen die drei Antirheumafaktorseren und gegen ein spezifisches Anti$\beta_{2}$-Makroglobulinserum eine identische Linie. Alle drei 